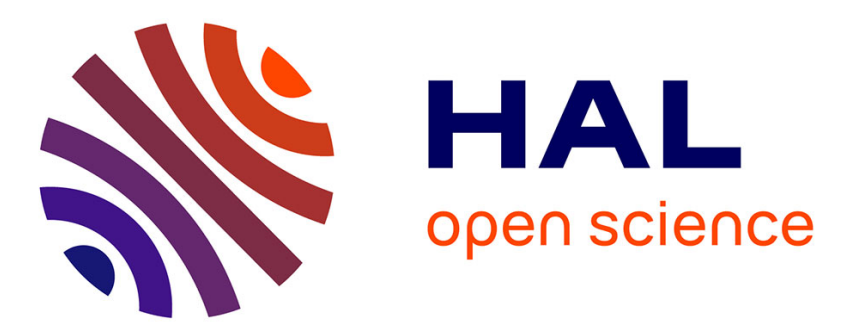

\title{
Considering human factors in BCI experiments: a global approach
}

\author{
Claudine Botte-Lecocq, Marie-Hélène Bekaert, Jean-Marc Vannobel, \\ Stéphanie Leclercq, François Cabestaing
}

\section{To cite this version:}

Claudine Botte-Lecocq, Marie-Hélène Bekaert, Jean-Marc Vannobel, Stéphanie Leclercq, François Cabestaing. Considering human factors in BCI experiments: a global approach. Journal Européen des Systèmes Automatisés (JESA), 2014, Méthodes, modèles et outils pour l'analyse et le contrôle de systèmes homme-machine, 48 (4-6), pp.283-301. 10.3166/jesa.48.283-301 . hal-01114440

\author{
HAL Id: hal-01114440 \\ https://hal.science/hal-01114440
}

Submitted on 17 Apr 2015

HAL is a multi-disciplinary open access archive for the deposit and dissemination of scientific research documents, whether they are published or not. The documents may come from teaching and research institutions in France or abroad, or from public or private research centers.
L'archive ouverte pluridisciplinaire HAL, est destinée au dépôt et à la diffusion de documents scientifiques de niveau recherche, publiés ou non, émanant des établissements d'enseignement et de recherche français ou étrangers, des laboratoires publics ou privés. 


\title{
Considering human factors in BCI experiments: a global approach
}

\author{
C. Botte-Lecocq ${ }^{1}$, M-H. Bekaert ${ }^{1}$, J-M. Vannobel $^{1}$, S. Leclercq ${ }^{2}$ \\ and F. Cabestaing ${ }^{1}$
}

\author{
1. LAGIS, Université Lille1, UMR CNRS 8219 \\ Cité scientifique - 59655 Villeneuve d'Ascq Cedex, France \\ Claudine.lecocq@univ-lille1.fr
}

2. CHRU Lille, France
plateformeNT@chru-lille.fr

RESUME. Le développement de nouveaux systèmes d'aide à la personne est l'une des applications interface cerveau ordinateur (BCI) les plus probables à court et moyen terme. Contrairement à de nombreuses technologies d'assistance récentes, les systèmes BCI actuels ne résultent pas d'une approche de conception universelle. De nombreux auteurs ont montré l'importance de prendre en compte des critères comme l'adaptabilité, la flexibilité, et autres critères relatifs à l'ergonomie lors de la conception d'une interface BCI. Dans cet article, nous proposons un cadre de travail visant à accélérer le transfert des expérimentations BCIs des laboratoires de recherche vers les services cliniques puis aux domiciles des patients. Plus précisément, nous montrons l'intérêt de concevoir une salle d'expérimentation dédiée (RBCIE) où les interfaces BCI peuvent être testées et validées efficacement.

ABSTRACT. The development of new enabling devices is one of the most credible short and medium-term applications of brain-computer interfaces (BCI). Unlike many other recent assistive technologies (AT), present BCI systems usually do not result from a "design for all" approach, since they are mainly effective for people suffering from severe motor disabilities. However, many authors have shown that it is crucial to take into account adaptability, flexibility, customisability and other ergonomics related criteria while designing a BCI-based AT. In this paper, we specify a framework that could expedite the transferability of BCI experiments from labs to clinical departments and later to patients' homes. We highlight the interest of designing dedicated experimentation facilities (RBCIE) in which the developed $B C I$ systems can be efficiently tested and validated. More generally, we highlight the interest of a multidisciplinary design approach introducing human factors at the core of concerns.

MOTS-CLES : Expérimentations BCIs, Conception centrée sur l'utilisateur, Ergonomie.

KEYWORDS: Assistive technology; Ergonomics; BCI experimentation; User centred design 


\section{Introduction}

A Brain-Machine Interface (BMI) aims at establishing a direct communication channel between user's brain and a man-made system. Several types of BMIs have been considered and experimented, such as neuroprostheses for restoring sensing capacities -- cochlear implants and more recently artificial retinas -- or motor capabilities (Velliste et al., 2008). In the last two decades, many research teams have focused their work on non-invasive Brain-Computer Interfaces (BCI) that allow a user to send commands to a computer through the recording and online processing of his EEG (electrical activity of the brain recorded along the scalp).

Whereas recent works have reported the use of non invasive BCIs for virtual reality control, entertainment and gaming (Allison et al., 2013; ), many BCI researchers stay focused on the development of BCIs assisting severely disabled people in maintaining or recovering autonomy. Thus, various experimental BCI systems (Bekaert et al., 2009 ; Mak and Wolpaw, 2009 ; Millán et al., 2010) can restore communication (Wolpaw et al., 2002) or mobility (Galán et al., 2008), allow object handling (Müller-Putz et al., 2005), control an artificial limb (Pfurtscheller et al., 2000 ; McFarland et al., 2008) or elements of the environment (Guger et al., 2008) and have also been regarded as a new therapeutic approach to rehabilitation (Dobkin, 2007 ; Van Langhenhove et al., 2008 ; Daly and Wolpaw, 2008).

Brain-computer interfacing is an interdisciplinary research field where many disciplines interact, such as neurobiology, neuroscience, computer and electrical engineering, robotics, ergonomics, cognitive psychology. Nevertheless, BCI research experiments and evaluation are usually described in the literature either from the technical or from the medical point of view. From the technical point of view, performance means accuracy and speed of signal processing and classification methods (Lotte et al., 2007 ; Bashashati et al., 2007). Performance can be evaluated through BCI competitions that "compare and select the best of breed in data analysis for EEG brain signals” (BBCI 2011). From the clinical point of view, performance evaluation implies clinical experiments involving people with severe motor disabilities, such as totally locked-in patients (LIS) who are unable to move or communicate due to a complete paralysis of all their voluntary muscles. In the last years, an increasing number of papers report experimental studies performed in a clinical framework on patients with severe neurological disorders (Cincotti et al., 2008, Kübler et al., 2013). Several teams have investigated whether or not disabled people can efficiently operate a BCI compared to healthy people (Silvoni et al., 2009 ; Ortner et al., 2011 ; Ang et al., 2011). Others have presented a methodology to measure individual user's ability to control a BCI-based Assistive Technology (AT) (Randolph and Moore, 2010 ; Allison and Neuper, 2010) or to adapt BCIs to individual users in order to expedite the technology-fit process. Indeed, while interaction and interfacing centred on the final user has already been widely studied in traditional Human Machine Systems (HMS), this issue is just being considered in BCIs (Tan and Nijholt, 2010, Allison et al., 2013). Even if the specificities of handicapped people are taken into account by BCI teams in their studies (Birbaumer et al., 2008 ; Parini et al., 2009 ; Nijboer and Broemann, 2010), human factors and 
ergonomic aspects in the experimental framework are not often reported (Nijboer et al., 2010 ; Kleih et al., 2010 ; Holz et al., 2013a).

Few BCI laboratories report on a successful long term use of non invasive BMIs at home by severely handicapped people (Birbaumer et al., 1999 ; Sellers et al., 2010 ; Holz et al. 2013b). Other teams report on experimentations in home environment in which a disabled patient has been excluded from the experimental protocol because the BCI system failed to detect a reliable feature in his input signals (Nijboer et al., 2008). It seems that a wide gap still exists between the development of BMIs inside research laboratories and the implementation of such systems at severely disabled patient homes. For an effective daily use by severely handicapped people with little or no supervising, BCIs must identify and adapt to the needs, wishes and abilities of potential users. Thus, several teams develop BCIs including more and more attractive HMIs such as the Virtual Reality cave-like system (Friedman et al., 2007), while others work on the development of universal BCIs (Allison, 2009 ; Miralles, 2010).

To cross the bridge in between, we propose to consider a dedicated physical experimentation space, namely a Room for BCI Experimentation (RBCIE), as a relevant tool for the development of BCIs just as simulators are standard tools for the development of traditional Human Machine Systems (Biswas and Robinson, 2007).

In this paper, we first show in which ways a RBCIE can help at the transferability of BCI systems to patient homes by dealing with their needs in real environment early in the development process. Then, we introduce a BCI user centred design according to a global quality approach which puts the patient at the centre of the concerns in accordance with four axes - needs, safety, well-being and effective everyday life purpose. In the following section, we detail how a RBCIE can highly contribute to the two last axes. We conclude on the actual need of focusing on ergonomic and human factors all along the design process, following a quality approach, in order to allow BCIs becoming ATs well adapted to severely impaired people.

\section{Why a RBCIE ?}

As the first phase of proof-of-concept is already done, we are now to the way on the second phase, the emulation one, where the developed technologies need to be tested and adapted (Mason et al., 2007), in order to commercialise them and to provide them to end-users in their daily life environment. For that purpose, we propose to perform experimentations in a RBCIE in order to analyse performance variability, to validate BCI systems and protocols, to assess the ecological validity of BCIs and to expedite the transferability of BCIs to patient homes by simulating daily life situations. 


\subsection{Analysis of variability}

When designing BCIs for handicapped people, one question is how and to which extent this BCI can generate an automatic control of a device while ensuring the reliability of the HMS. The performance variability of the HMS depends on three main factors: the user (inter and intra individual differences), the experimental framework and the activity (Jacko, 2003). The user variability is mainly due to attention problems, postures, bio-mechanical and physiological constraints, problem solving difficulties, sensory perception disorders and stress management. The experimental framework variability is directly related to the protocol itself and to the experimental conditions. The activity variability is mainly due to its mental aspect.

A RBCIE has to isolate individual sources of variability and study how they interact with each other. For example, the physiological patterns used in BCIs show a significant variation from one user to another (Schreuder et al., 2013). To analyze this user variability, it is necessary to fully control the experimental framework and to precisely describe the required mental activity. A RBCIE ensures this control all along the BCI design phase thanks to fully mastered environment and protocol.

In the same way, it is important to introduce everyday life environmental perturbations during experiment sessions and to check the stability of experimental conditions in order to ensure the reliability and the robustness of the BCIs. A RBCIE enables extensive experimentation over long time periods and in various environmental situations. It is crucial to assess the effect of variability on BCI performance (Gowreesunker et al., 2011).

The mental activity required to generate a discriminating pattern, necessary to control the device, is often abstract (hand moving, counting ...). In this regard, each user can settle different scenarios (pressing a ball, drumming fingers, scratching, counting one by one, seven by even, starting at 0 or not, ...). So, for a given user, in constant environmental conditions, the RBCIE enables to measuring the impact of the mental activity variability on the HMS performances. Consequently, it enables to select the optimal mental scenario for a given purpose for each user.

In real life situations, the three variabilities are concomitant. In the RBCIE, the 3 variabilities can be analysed separately but also combined. This ensures the robustness of the designed BCI.

\subsection{Validate BCI systems and protocols}

The BCI experimentations can be settled either to approve a protocol, to confirm some behaviour hypothesis, to validate models, tools or new BCI communication paradigms. Thus, a RBCIE could be usable for different experimentations intended for various recipients (patients with different disabilities, neuroscientists, engineering researchers, psychologists, linguists) with different objectives and sometimes performed with an incomplete knowledge of the overall issue. In any case, a lot of data need to be collected including those measuring the behaviour of 
the subject, the activity of all the people involved in the experiment (subject, experimenter, caretaker), the ambience parameters and the activity conditions. That alone justifies the interest of a specifically equipped space such as a RBCIE.

\subsection{Assess the ecological validity of BCIs}

Since BCI-based AT are mainly intended to assist disabled people, not only in a clinical context but also at home, the ecological validity of the BCI carried out have to be assessed. When experimentations are settled in engineer science laboratories, BCIs are often validated by means of experimentations in highly controlled environmental conditions using healthy users (students, researchers, engineers) who do not have necessarily the same needs, expectations, and practices as disabled people. This can lead to a low ecological validity particularly when cognitive processes are involved (Kerick and Mcdowell, 2009). The use of a RBCIE could expedite the evaluation of the BCI in a more or less controlled ecology. Indeed, two additional issues are related to the non perceptive nature of BCIs. First, as brain activity is not a communication channel between people, neither the interaction between the subject and the BCIs nor the subject command understanding are not directly observable. Second, the evaluated BCI is at the same time the object of evaluation and the evaluation tool. With respect to these issues, a RBCIE could help at developing specific evaluation tools. Moreover, most of BCI applications for severely handicapped people only consider data coming from the mental activity. To improve the reliability of the BCIs, to make this new technology suitable for less disabled people, but also to expedite the usability evaluation of the BCIs, the RBCIE could be equipped with sensors to acquire human senses information such as vision, auditory and feel as well as brain activity and physiological data. Thus, more and more researchers of the BCI community develop hybrid BCIs involving either several brain activity sources (pure hybrid BCIs), or a brain activity combined with a physiological one (physiological hybrid BCI) or a brain activity and another input (mixed hybrid BCI) (Pfurtscheller et al., 2010 ; Amiri et al., 2013).

\subsection{Expedite the transferability of BCIs to patient homes by simulating daily}

\section{life situations}

Home BCI implementation is difficult for many different reasons. Indeed, the equipment comprising electrodes, caps, amplifier, and computers is neither plug and play nor easy to use. It requires time to be set up as well as enlightened caretaker assistance. The BCI system needs a continuous technical support to regularly update the system parameters to calibrate the system or to intervene in case of system failure. It can need long training periods, sometimes months (Birbaumer, 2006) and the communication rate is rather slow (one to three characters per minute in case of spellers operated by handicapped people). Moreover, it requires a continuous controlled attention and is very emotional energy consuming to communicate with the system for a long period of time. 
One of the advantages of supervised experiments, namely in vitro experiments, is to enable high reproducibility of experimental conditions and to simulate complex, unusual or critical situations (Davis et al., 2009 ; Nam et al., 2010). In addition, maintenance problems and remote system management could be settled before the home transfer.

But, to expedite the transferability of BCIs to patients' homes, BCI research must still put a lot of emphasis on real-world experimentations with disabled people (Vaughan et al., 2006). The simulation of home-like environments, namely in vivo experiments, in a modular RBCIE would enhance the usability of the BCIs by simulating situations more or less close to everyday life conditions.

\section{A global approach to BCI systems design}

Since 2007, we mostly focus our research activity on the development of palliative communication interfaces. First of all, we developed a portable system capable of quickly adapting to the patient by means of screening data (Van Langhenhove et al., 2008). Then, in order to transfer lab experiments to clinical use, we aimed at defining the most adapted and ergonomic experimental framework for the patients by collaborating with a rehabilitation engineer specialised in new assistive technologies (occupational therapist) (Leclercq et al., 2010). This collaboration did encourage us, as others researchers (Cincotti et al., 2008 ; Parini et al., 2009), to consider the usability of BCIs for less severely handicapped people such as patients suffering from the Duchene Muscular Dystrophy (DMD), a progressive disease resulting in muscle degeneration, difficulty walking, difficulty breathing, and death. There is a double benefit to open BCI-based AT to these patients since traditional communication channels are still standing for a while. First, the patient progressively adapts to the technology and the tool can be progressively adapted to the evolution of the disease. As an example, Birbaumer (2006) related that none of the 7 ALS patients who were trained to their SCP-BCI after entering the complete locked-in state were able to achieve a lasting BCI control and communication. On the opposite, 7 ALS patients who were trained before entering in the locked-in state were capable of continuing to use the BCI afterwards. As a second benefit, the patient can be included in the BCI design process thanks to the remaining communication channels. In this way, researchers involved in the BRAIN project develop user-centred methods focusing on a direct involvement and engagement of the final user within both design and evaluation of the BCI systems (Gräser, 2008)

The different approaches to User-Centred Design (UCD) are based a lot on the international standard ISO 9241-210/2010 (Human Centred Design for Interactive Systems). This standard defines a general process for including human-centred activities throughout a development life-cycle composed of four groups, namely the specification of the context of use, the specification of the user requirements, the creation of the design solution, and the evaluation of the design (Buurman, 1997). It is now acknowledged that UCD optimizes the fit and leads to more usable systems, especially in systems that involve human-computer interaction or software. The 
involvement of end-users is particularly important in the case of assistive products as the designers are usually not themselves disabled and are therefore unlikely to be aware of these requirements (Bradley and Dunlop, 2008).

This upstream collaboration between researchers and the final users led us to follow a quality approach (Figure 1) inspired by the PDSA (Plan Do Study Act) cycle defined by Deming (Moen and Norman, 2006) which is de facto user-centred. This approach is a cyclic process for planning, formalising and testing improvement activities all along the product life-cycle. Such a cycle induces a continuous improvement of the quality. Now, it implies a product not only with high technical performance but also easy to use and fitting with user practices and activities. Software quality in ISO/IEC 9126 standard is categorised from a user perspective as functionality, reliability, usability, efficiency, maintainability and portability (Bevan, 1999).

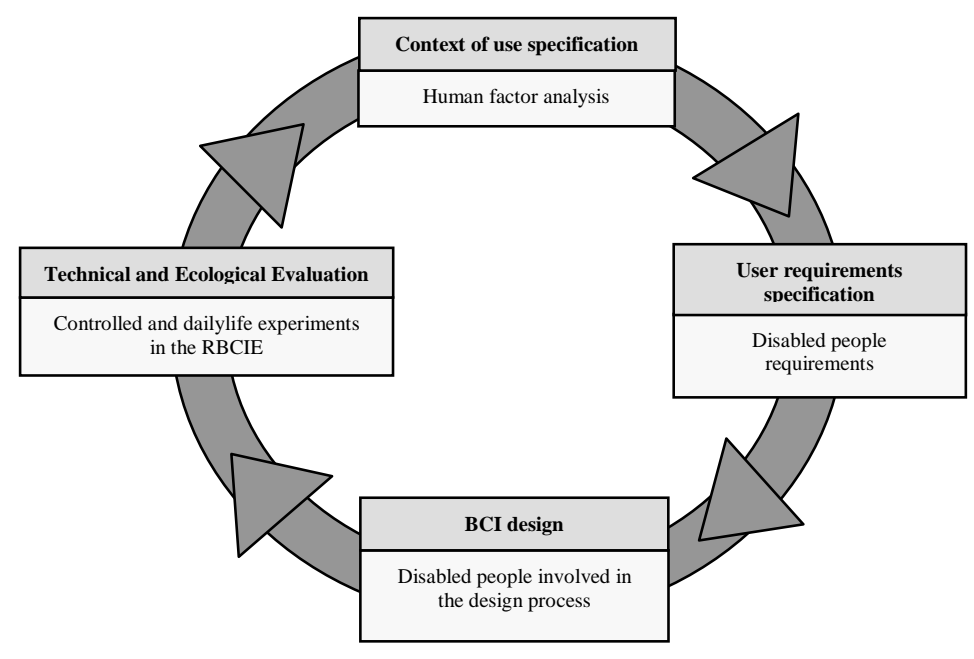

Figure 1. BCI User-Centred Design according to a Quality Approach

Considering a BCI UCD according to a quality approach logically associates the Plan phase with the analysis of human factors and interactions involved in the BCI system and with disabled user requirements. The Do phase is then associated with the user-centred design itself. The disabled patient is involved as a protagonist of the design as soon as his handicap allows it. The Study phase is associated with BCI technical and ecological evaluation by performing experiments in a more or less controlled environment inside the RBCIE. Finally, the Act phase consists in improving the BCI system towards the evaluation results. Thus, the BCI design process is part of a continuous loop so that researchers can identify and change the parts of this process that need improvements. This iterative process enables adaptations to the individual characteristics and then avoids focusing on the limits of the device or on the experimenter's skills. 
This quality approach puts the patient at the centre of the concerns in accordance with four main aspects: his needs, his well-being, his safety, and an effective everyday life purpose (Figure 2).

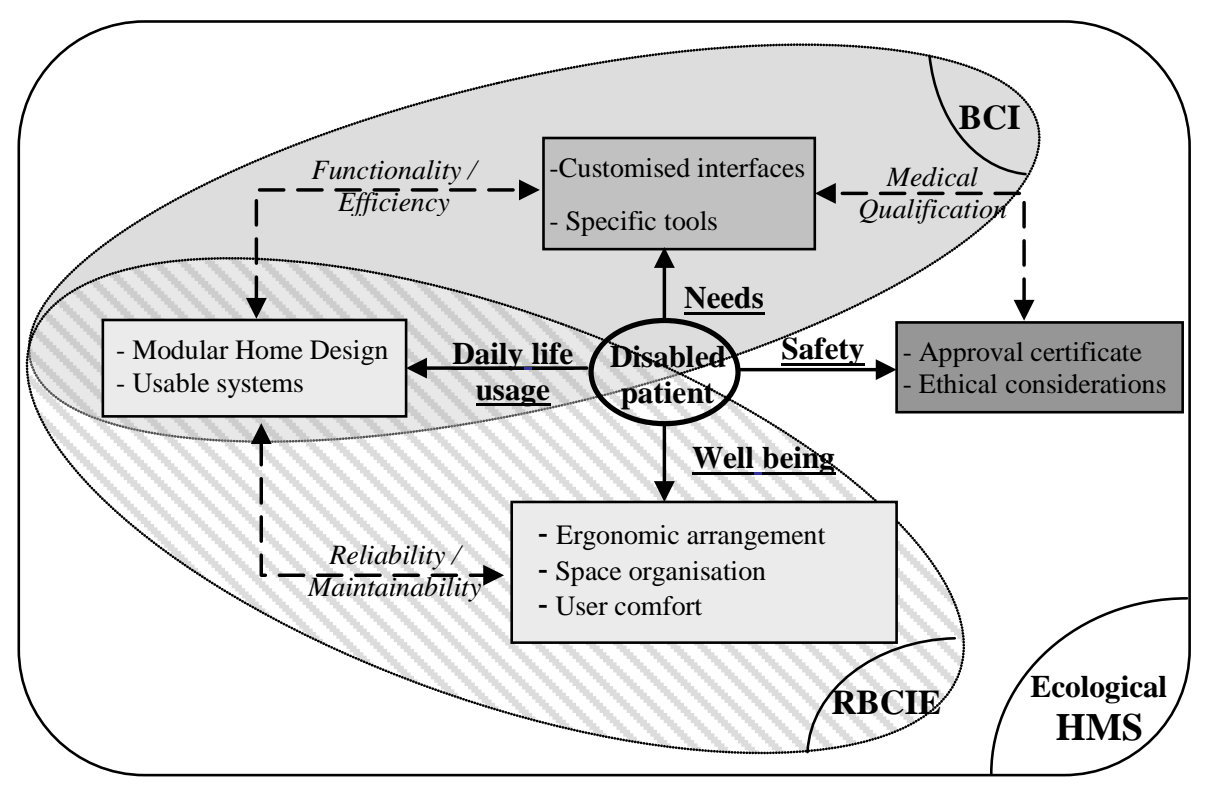

Figure 2. Existing BCI systems (grey ellipse) - RBCIE contribution to BCI systems (shaded ellipse)

It is important to take into account the patient well being and needs with respect to his handicap or his disease specificities in BCI systems design as well as in the RBCIE one. Accessibility, ergonomic arrangement, space organisation, user comfort are concerned human factors to focus on in order to improve the system dependability. The RBCIE design must be based on existing guidelines on EEG biomedical departments (Malkin, 2002 ; Binnie et al., 2003 ; ) and on ergonomic space layout practices (Letho and Buck, 2008) in compliance with accessibility national or international standards or regulation (JORF, 2005 ; Federal Register, 2004) and of course in compliance with national regulations and laws. In France, designing a RBCIE requires a building agreement by the DRASS to insure that facilities are well suited to research, and compatible with people safety. Many conditions have to be checked: the building has to be compliant with the standard, a safety cabinet with emergency devices such as oxygen units, or defibrillators must be quickly accessible, and an emergency procedure must be well-defined.

As BCI research mainly deals with patients needs by developing specific tools for communication and control, mobility assistance, rehabilitation and entertainment, they are mainly evaluated in terms of functionality and efficiency 
(Millán et al., 2010). A systematic emphasis on other needs such as userfriendliness, easiness of use, aesthetic (Münßinger et al., 2010), would lead to more generic and intuitive interfaces customisable by the final user according to his own desires and needs (Gräser, 2008). Experimenting these systems in a modular RBCIE in home-like situations, including environment perturbations scenarios, would increase their maintainability and reliability while improving their usability. Finally, the conformity assessments of the medical devices as well as ethical considerations have to be regarded to ensure the safety of the patient (Tamburrini, 2009 ; Grübler et al., 2013).

\section{USER-CENTRED CONSIDERATIONS: RBCIE CONTRIBUTION TO BCI SYSTEMS DESIGN}

\subsection{Well Being}

\subsubsection{Accessibility: markings and facilities}

Around the RBCIE, an environment suitable for both motor-disabled and ablebodied people has to be thought. Accessibility needs to be considered from a global point of view taking into account different kinds of disabilities and different profiles of users. Generally speaking, resting facilities, ramps, parking spaces and access are needed to make area access easier. Mobility impairments which involve the use of wandering assistance (walking assistance, wheelchair, scooter, walker frames...) will affect space allowance and clutter. Grasping areas need to be set up according to the disabled capabilities to reach door handles, digital locks, entry phones or light switches. Auditory or visual impairments lead to locate visual, tactile or kinaesthetic signage at specific places. It also leads to use realistic pictures to mark the different spaces and their functions. Whatever the signage, the most important is its efficiency and immediacy (Preisez and Smith, 2010).

These accessibility constraints highlight the inherent difficulties to implement a RBCIE out of a clinical environment, even if the non-medical buildings where the facilities are installed are often public ones and therefore gradually standardised according to the legal requirements for example, the "Equality and Handicap 2005" law in France).

\subsubsection{Space organisation and ergonomic arrangement}

Organising the space consists in first defining the parts dedicated to the different participants, their communication channels and the circulation loop and then dealing with space fitting.

In accordance with the literature (Cincotti et al., 2008), it seems relevant to split the RBCIE into two distinct well-identified spaces: one observation space dedicated to experimenters to control the whole experiment, and one experimentation space dedicated to the subject. In the experimentation space, a manoeuvring clearance space and an ergonomic workspace are at least required. The observation space will 
be organised such as to define an ergonomic workplace for the experimenter, a welcome place for the subject, a waiting space for the caretaker, and a clearance space (Figure 3).

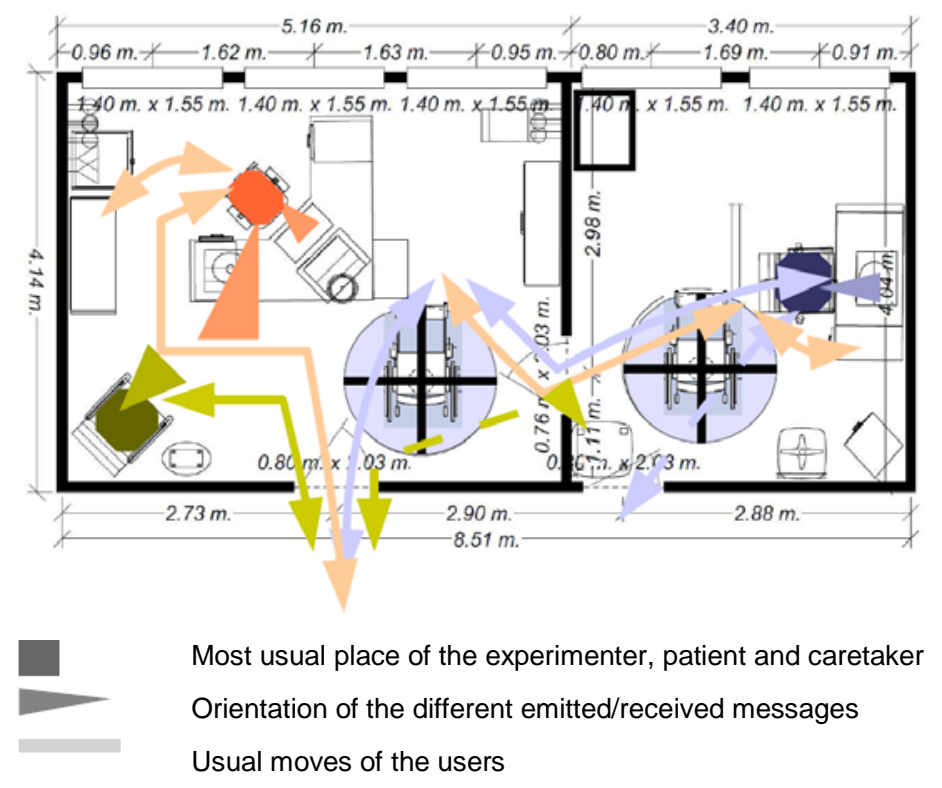

Figure 3. Space fitting and circulation of all the protagonists inside the RBCIE

Two kinds of circulation areas into or between spaces can be defined in the circulation loop: frequently passing spaces and critical areas where several persons have to meet at the same time. Clear widths of each space must be large enough for disabled people movements. Complying with anthropometric data standards ensures enough space for able-bodied as well as for disabled people to move freely and safely (ISO 7250).

Space fitting must be optimised, secured, adapted to everyone and identically reconfigurable from session to session. To relevantly place furniture and BCI equipments, it is important to notably worry about workspace brightness, desk orientation keeping in mind circulation and communication between protagonists (Corlett and Clark, 2009). All elements need to be set through the spaces depending on their bulk and their frequency of use such as to reduce awkward postures or moving constraints and to extend time-saving (ISO 6385).

Finally, it is important to organise space while thinking about people communication. With disabled people, the direct communication channels can be either verbal or written ones, or using a keyboard combined with a speech synthesis. Indirect channels carrying the message during experiments can be microphones 
combined with loudspeakers in both spaces. Video surveillance equipment as well as bay-windows can help to make sure of subject well-being, stillness and safety. Indirect communication channels can also be used to carry warning signals from the subject to the experimenter.

\subsubsection{User comfort}

For both experimenters and users, a BCI experiment in a RBCIE can be viewed as a workplace where work periods, characterised by their physical fatigue effects, their duration, accuracy and repetitiveness, are punctuated by rest periods during which energy expense is minimised. Work usually implies complex and simple gestures as well as working postures, and requires some mental workload inducing intellectual and/or emotional constraints and intellectual fatigue. Studies have shown that the design and ambience (lighting, thermal, sound) of working spaces affect user comfort, motivation, stress tolerance and well-being and that these factors impact on error and accident rates, productivity and work quality.

Veitch et al. (2008) have shown that, when given control over their lighting, workers will vary their lighting choices according to the tasks they are doing in order to maximize their performance. Workers who were satisfied with their lighting considered the space to be more attractive, were happier, more comfortable and more satisfied with their environment and their work ((Newsham et al., 2004).

Thermal comfort, defined as the state of mind that expresses satisfaction with the surrounding environment has also an impact on user performance (Van Hoof et al., 2010). ISO/TS 14415/ 2005 Standard provides criteria and methods for the assessment of the thermal comfort of the disabled.

Noise also has effects on stress and mental performances (Belojevic et al., 1992). Wall, floor or ceiling absorbent materials as well as an appropriate partitioning of furniture across spaces can restraint the different noise sources and their reverberations.

Colours also affect minds. Except for safety elements, choosing cool, sober, soothing colours primarily helps keep people mind more serene, and secondly avoids eye strain caused by strong contrasts (Stone and English, 1998).

\subsubsection{User mental comfort}

The user's mindset highly impacts on the experimentation success. Nevertheless, stress factors can be limited by focusing on the subject well-being all along BCI experiment sessions making his disability adaptation process easier as well as his psychological acceptance (Louise-Bender et al., 2002). Indeed, the experimenter can highly contribute to make the subject feel secure all along the experiment. People management factors such as consistency, respect, inclusion, trust and honesty have a decisive influence on experimentation sessions. Therefore, the sessions have to be adapted to the needs and the difficulties of the subject regarding these factors which implies consideration to the subject and respect of the differences. During sessions, if the experimenter totally masters both room spaces and BCI system, he has no 
mental overload and can focus on the human aspect (discussing, reassuring, and inducing a mutual confidence feeling). To ascertain the experimentation feasibility, a meeting, well before the experimentation itself, leads to reduce the experiment duration and avoid any subject disappointment. Thus, the experimenter may check the most important aspects to discuss with the subject prior to the experiment, and make sure of his well-understanding. The patient will feel reassured if he is informed that he may stop the experiment whenever he wants. At the end of this meeting, a written protocol, as well as an informed consent form, is signed by the patient to well define the performed study and the expected results, and also to guarantee the experiment reproducibility and then the comparison of the results. A relatively extendable time can be devoted at the beginning of the experiment to introduce the RBCIE environment and equipment to the subject according to his stress tolerance and his understanding capabilities. This will help the subject to feel unworried, relaxed and at ease. Then, during the recordings, the most guided and coercive phase of the experiment, the protagonists are very much in demand which requires optimum concentration. At the end of the experiment, an interview phase can also help the subject to feel confident. During this step, the experimenter can yield an experience background to the subject, and can write down the expressed subject feelings and his expected improvements.

\subsection{Daily life usage}

Nowadays, one of the main challenges in BCIs is to go out of the laboratories. A halfway solution is to move towards in vivo experiments in a RBCIE where subjects can proceed more or less freely while experimenters observe subject and device behaviour. In this perspective, a modular home design of the RBCIE will help to highlight interactions, to analyse required constraints and skills and to understand disability (Quek et al., 2011).

\subsubsection{Modular home design}

To experiment BCI systems before their transfer to patient homes, the RBCIE can be fitted out with household furniture and equipments to validate the BCI in quasi-real conditions. It is thus possible to artificially generate and control environmental perturbations to measure their influence on the system by properly equipping the RBCIE. Television or radio background noise, sudden light variation or verbal interjection can be easily generated. The daylight lighting can be controlled by window blinds. Thermal conditions can be controlled by fan equipments, air-conditioning or thermostatic-based heating systems. In vivo experimentations can highly contribute to the reliability and robustness improvement of the BCIs before their transfer to patient homes. In addition, it is possible in such a facility to set up failure scenarios in safety conditions to study the subject and system behaviours in such situations. Moreover, setting in vivo experiments in a RBCIE can contribute to set up optimal maintenance and updating procedures such as to make the system as user transparent and automatically controlled as possible. 


\subsubsection{Usable systems}

According to ISO 9241-11, usability is defined as "The extent to which a product can be used by specified users to achieve specified goals with effectiveness, efficiency, and satisfaction in a specified context of use". The interface usability is defined in the literature according to its efficiency of use, the user subjective satisfaction, its easiness of learning or remembering and its error rate. Thus, Nielsen (1993) defines ten heuristics for guiding the evaluation of HMI system usability while Bastien and Scapin (1992) suggest eight heuristics. Most of those heuristics can be matched. However, the first author emphasizes the error aspect so that his criteria mainly aim at resolving them. The second author focuses more on adaptability and learning possibility.

Most of these usability criteria, developed for perceptive HMS, are relevant for non perceptive HMS with pattern recognition such as BCIs. Nevertheless, some of these critreria need to be readjusted in regard with BCI specificities. First, the mental workload of the subject associated to a particular task needs to be strong enough to force him to concentrate on only one task at a time to facilitate the detection of the associated mental state. It seems thus important to readjust the standard comfort zone related to the mental workload, not only to prevent the emergence of interferences but also to avoid the dilution of the signal in the noise while limiting the mental fatigue of the subject. Second, the match between system and the real world (compatibility criterion) requires the HMS to be compatible with the human beings and their habits. Affordance which is the ability of an object to suggest its own use is the pinnacle of human compatibility. In BCIs, this criterion is sometimes disregarded to the benefit of a discriminative efficiency of the patterns generated by the subject in terms of reliability and response time (Millan, 2003 ; Sepulveda et al, 2007 ; Bos et al., 2011). Thus, mental tasks are often far away from the actions usually performed by the subject such as a word association task to move a wheelchair (Galan et al., 2008). Third, in view of the flexibility and efficiency of use (adaptability criterion) relevant to BCIs, the system must adapt to the user, particularly to his individual characteristics, his novice or expert status, and his impairment. This criterion is crucial for BCIs because of many intra-subject and inter-subject's differences. Fourth, the BMI control by the user is highly related to the feedback which provides information on what the system is able to translate as the users' intent. This interpreted feedback is currently not synchronized with the user corrective and decisional process (Trejo et al., 2006).

Through in vivo experiments, the RBCIE can highly contribute to measure BCI systems usability, specifically when this latter has to be quantified by means of indirect measures or attributes such as the number of reported problems with easeof-use, aesthetic, and comfort all along the usage.

\section{Conclusion}

In the literature, many authors relate the crucial need of developing BCIs while taking into account adaptability, autonomy, training methods, environment artefact 
control, functionality, and aesthetics. This paper shows that human factors have to be the major concerns while designing BCI-based AT. To insure the ecological validity of such systems, it is essential to follow a user-centred design method involving the final user early in the design, and to experiment and evaluate BCIs with disabled people in daily life conditions. Thus, a dedicated and ergonomically designed room called RBCIE can help the research teams to reach this goal.

Testing the usability of already developed BCI systems through in vivo experiments would really help identify the applications transferable to the patient home with respect to his needs. This implies not only more experiment sessions with handicapped people, but also more assessments, more reviews and more feeling collection.

\section{Bibliographie}

Allison B. (2009). The I of BCIs: next generation interfaces for brain-computer interface systems that adapt to individual users, Human-Computer Interaction, vol. 2, p. 558-568.

Allison B., Neuper C. (2010). Could Anyone Use a BCI?, Applying our Minds to HumanComputer Interaction, D.S. Tan and A. Nijholt eds, Human-Computer Interaction Series, Springer, p. 35-51.

Allison B.Z., Dunne S., Leeb R., Millán JdR., Nijholt A. (2013). Towards Practical Brain-Computer Interfaces: Bridging the Gap from Research to Real-World Applications, Biological and Medical Physics, Biomedical Engineering Spinger (Ed)

Amiri S., Fazel-Rezai R., Asadpour V. (2013). A Review of Hybrid Brain-Computer Interface Systems.Advances in Human-Computer Interaction,Vol.2013

Ang K.K., Guan C., Chua K.S., Ang B.T., Kuah C., Wang C., Phua K.S., Chin Z.Y., Zhang H. (2011). A large clinical study on the ability of stroke patients to use EEG-Based motor imagery Brain Computer Interface, Journal of Clinical EEG \& Neuroscience, vol. 42, n 4 , p. 253-258.

Bashashati A., Fatourechi M., Ward R.K., Birch G.E. (2007). A survey of signal processing algorithms in brain-computer interfaces based on electrical brain signals, Journal of Neural Engineering, vol. 4, n², R32-R57.

Bastien J.-M., Scapin D.L. (1992). A validation of ergonomic criteria for the evaluation of human-computer interfaces, International Journal of Human Computer Interaction, vol. 4, p. 183-196.

BBCI 2011, Berlin Brain-Computer Interface. Available from: http://www.bbci.de /competition/.

Belojević G., Öhrström E., Rylander R. (1992). Effects of noise on mental performance with regard to subjective noise sensitivity, International Archives of Occupational and Environmental Health, vol. 64, n4, p. 293-301.

Bekaert M.-H., Botte-Lecocq C., Cabestaing F., Rakotomamonjy A. (2009). Les interfaces Cerveau-Machine pour la palliation du handicap moteur sévère, STH Sciences et Technologies pour le Handicap, vol. 3, n¹, p. 95-121. 
Bevan N. (1999). Quality in use: meeting user needs for quality, Journal of Systems and Software, vol. 49, $\mathrm{n}^{\circ}$ 1, p. 89-96.

Binnie C., Cooper R., Mauguiere F., Osselton J., Prior P., Tedman B. (2003). Clinical Neurophysiology: EEG, paediatric neurophysiology, special techniques and applications, Elsevier Health Sciences eds.

Birbaumer N., Ghanayim N., Hinterberger T., Iversen I., Kotchoubey B., Kubler A. (1999). A spelling device for the paralysed, Nature, vol. 398, p. 297-298.

Birbaumer N. (2006). Breaking the silence: Brain-computer interfaces (BCI) for communication and motor control, Psychology, vol. 43, p. 517-532.

Birbaumer N., Murguialday A.R., Cohen L. (2008). Brain-computer interface in paralysis, Current Opinion in neurology, vol. 21, n 6, p. 634-638.

Biswas P., Robinson P. (2007). Simulation to predict performance of assistive interfaces, Proceedings of the 9th International ACM SIGACCESS Conference on Computers and Accessibility, ASSETS'07, Arizona.

Bos D. P-O., Poel M., Nijholt A. (2011).A Study in User-Centered Design and Evaluation of Mental Tasks for BCI. Advances in Multimedia Modeling, Lecture Notes in Computer Science, vol. 6524, p. 122-134

Bradley N., Dunlop M. (2008). Navigation AT: context-aware computing, Assistive technology for visually impaired and blind people, M.A. Hersh and M.A .Johnson eds, Springer Verlag, p. 231-260.

Buurman den R. (1997). User-centred design of smart products, Ergonomics, vol. 40, n 10, p. 1159-1169.

Cincotti F. Mattia D., Aloise F., Bufalari S., Schalk G., Oriolo G., Cherubini A., Marciani M.G., Babiloni F. (2008). Non-invasive brain-computer interface system: towards its application as assistive technology, Brain Research Bulletin, vol. 75, n6, p. 796-803.

Corlett E.N., Clark T.S. (2009). The ergonomics of workspaces and machines: a design manual, Second Edition, Taylor\&Francis.

Daly J., Wolpaw J. (2008). Brain computer interfaces in neurological rehabilitation, The Lancet Neurology, vol. 7, n¹1, p. $1032-1043$.

Davis G., Popovic D., Johnson R.R., Berka C., Mitrovic M. (2009). Building Dependable EEG Classifiers for the Real World - It's Not Just about the Hardware, Proceedings of the 5th Int. Conference on Foundations of Augmented Cognition. Neuroergonomics and Operational Neuroscience: Held as Part of HCI International 2009 (FAC '09), p. 355364.

Dobkin B.H. (2007). Brain-computer interface technology as a tool to augment plasticity and outcomes for neurological rehabilitation”, Journal of physiology, vol. 579, n³; p. 637-642.

Federal Register. (2004). ADA and ABA Accessibility Guidelines for Buildings and Facilities

Friedman D., Leeb R., Guger C., Steed A., Pfurtscheller G., Slater M., (2007). Navigating virtual reality by thought : What is it like?, Presence-Teleoperators and Virtual Environments, vol. 16, ${ }^{\circ} 1$, p. 100-110. 
Galán F., Nuttin M., Lew E., Ferrez P.W., Vanacker G., Philips J., Millán JdR. (2008). A Brain-Actuated Wheelchair: asynchronous and non-invasive Brain-Computer Interfaces for continuous control of robots, Clinical Neurophysiology, vol. 119, p. 2159-2169.

Gowreesunker B.V., Tewfik A.H., Tadipatri V.A., Ashe J., Pellize G., Gupta R. (2011). A Subspace Approach to Learning Recurrent Features From Brain Activity, IEEE Transactions on Neural Systems and Rehabilitation Engineering, vol. 19, n ${ }^{\circ}$ 3, p. $240-$ 248.

Gräser A., (2008). Brain-computer interfaces with Rapid Automated Interfaces for Nonexperts, EU-project BRAIN (ICT-2007-224156), 2008-2011.

Grübler G., Al-Khodairy A., Leeb R., Pisotta I., Riccio A., Rohm M., Hildt E.(2013). Psychosocial and Ethical Aspects in Non-Invasive EEG-Based BCI Research-A Survey Among BCI Users and BCI Professionals. Neuroethics, February 2013

Guger C., Holzner C., Grönegress C., Edlinger G., Slater M. (2008). Control of a Smart Home with a Brain-Computer Interface, www.gtec.at/content/ download/1825/11409 /version/2.

Holz E., Zickler C., Riccio A., Höhne J.,Cincotti F., Tangermann M., Halder S., Mattia, D., Kübler A. (2013a). Evaluation of Four different BCI prototypes by severely motorrestricted End-Users, Proceedings of the Fifth International Brain-Computer Interface Meeting 2013

Holz E., Botrel L., Kübler A. (2013b). Bridging Gaps : Long-Term Independent BCI Home-Use by a Locked-In End-User, Proceddings of TOBI Workshop IV, Sion, Switzerland.

Jacko J.A., Stephanidis C., Duffy V. (2003). Human-Computer Interaction - Theory and Practice, CRC Press, vol. 3, Routledge.

JORF. (2005). Loi N 2005-102 pour l'égalité des droits et des chances, la participation et la citoyenneté des personnes handicapées (2005), JORF n³6 2005.

Kerick S.E., Mcdowell K. (2009). Understanding Brain, Cognition, and Behavior in Complex Dynamic Environments, Proceedings of the 5th Int. Conference on Foundations of Augmented Cognition. Neuroergonomics and Operational Neuroscience: Held as Part of HCI International 2009 (FAC '09), p. 35-41.

Kleih S.C., Nijboer F. Halder S., Kübler A. (2010). Motivation modulates the P300 amplitude during brain-computer interface use, Clinical Neurophysiology, vol. 121, $\mathrm{n}^{\circ}$, p. 1023-1031.

Kübler A., Holz E., Kaufmann T., Zickler C. (2013). Brain-Computer Interface Systems Recent Progress and Future Prospect, Fazel-Rezai R. Editor, Published by InTech.

Leclercq S., Bekaert M-H., Botte-Lecocq C., Cabestain F. (2010). Focusing on Human Factors while Designing a Brain Machine Interface (BMI) Room, AMSE-journals, Modelling Measurement and Control, vol. 2010, p. 92-99.

Letho M.R., Buck R. (2008). Introduction to human factors and ergonomics for engineers, Human factors and ergonomics, Gavriel Salvendy Series eds.

Lotte F., Congedo M., Lécuyer A., Lamarche F., Arnaldi B. (2007). A review of classification algorithms for EEG-based brain-computer interfaces, Journal of Neural Engineering, vol. 4, n², R1-R13. 
Louise-Bender P.T., Kim J., Weiner B. (2002). The shaping of individual meanings assigned to assistive technology: a review of personal factors, Disability and Rehabilitation, vol. 24, $\mathrm{n}^{\circ} 1 / 2 / 3$, p. 5-20.

Mak J., Wolpaw J. (2009). Clinical Applications of Brain-Computer Interfaces: Current State and Future Prospects, IEEE Reviews in Biomedical Engineering, vol. 2, p. 187-199.

Malkin J. (2002). Medical and dental space planning: a comprehensive guide to design, equipment, and clinical procedures, John Wiley and Sons eds.

Mason S., Bashashati A., Fatourechi M., Navarro K., Birch G. (2007). A comprehensive survey of Brain Interface Technology Designs, Annals of Biomedical Engineering, vol. 35, n², p. 137-169.

McFarland D., Sarnacki W., Wolpaw J., (2008). Electroencephalographic (EEG) control of three-dimensional movement, Journal of Neural Engineering, vol. 7, n³.

Millán, JdR., Rupp R., Müller-Putz G., Murray-Smith R., Giugliemma C., Tangermann M., Kübler A., Leeb R., Neuper C., Müller K. R., Mattia D. (2010). Combining braincomputer interfaces and assistive technologies: state-of-the-art and challenges, Frontiers in Neuroscience, vol. $4, n^{\circ} 161$.

Miralles F. (2010). Autonomy and social inclusion through mixed reality Brain-Computer Interfaces: Connecting the disabled to their physical and social world. EU-project BrainAble, 2010-2012.

Moen R., Norman C. (2006). Evolution of the PDCA cycle, http://www.pkpinc.com/files/ na01moenNormanfullpapers.pdf

Müller-Putz G., Scherer R., Pfurtscheller G., Rupp R. (2005). EEG-based neuroprosthesis control: A step towards clinical practice, Neuroscience letters, vol. 382, p. 169-174.

Nam C.S., Li Y., Johnson S. (2010). Evaluation of P300-Based Brain-Computer Interface in Real-World Contexts, International Journal of Human-Computer Interaction, vol. 26, $n^{\circ} 6$, p. 621-637.

Newsham G., Veitch J., Arsenault C., Duval C. (2004). Lighting for VDT Workstations 2: Effect of Control and Lighting Design on Task Performance, and Chosen Photometric Conditions, National Research Council of Canada, Institute for Research in Construction.

Nielsen J. (1993). Usability engineering, M. Kaufmann eds, Academic Press, United States.

Nijboer F., Sellers E.W., Mellinger J., Jordan M., Matuz T., Furdea A., Halder S., Mochty U., Krusienski D.J., Vaughan T.M., Wolpaw J.R., Birbaumer N., Kübler A. (2008). A P300-based brain-computer interface for people with amyotrophic lateral sclerosis, Clinical Neurophysiology, vol. 119, n8, p. 1909-1916.

Nijboer, F., Broermann U. (2010). Brain-Computer Interfaces for communication and control in locked-in patients, Brain-Computer Interfaces_Revolutionizing HumanComputer Interaction, B. Graimann, B. Allison and G. Pfurtscheller eds, The Frontiers Collection, Springer, p. 185-202.

Nijboer F., Birbaumer N., Kubler A. (2010). The influence of psychological state and motivation on brain-computer interface performance in patients with amyotrophic lateral sclerosis - a longitudinal study, Frontiers in Neuroscience, vol. 4, n55. 
Ortner R., Aloise F., Prückl R., Schettini F., Putz V., Scharinger J., Opisso E., Costa U., Guger C. (2011). Accuracy of a P300 Speller for people with motor impairments: a comparison, Journal of Clinical EEG \& Neuroscience, vol. 42, n 4, p. 214-218.

Parini S., Maggi L., Turconi A., Andreoni G. (2009). A Robust and Self-Paced BCI System Based on a Four Class SSVEP Paradigm: Algorithms and Protocols for a HighTransfer-Rate Direct Brain Communication, Journal Computational Intelligence and Neuroscience, vol. 2009, ID. 864564.

Pfurtscheller G., Guger C., Müller G., Krausz G., Neuper C. (2000). Brain oscillations control hand orthosis in a tetraplegic, Neuroscience Letters, vol. 292, n³3, p. 211-214.

Preisez W., Smith K. (2010). Universal Desingn Handbook, 2E, Mc Graw Hill Professionnal.

Quek M., Boland D., Williamson J., Murray-Smith R.,1 Tavella M., Perdikis S., Schreuder M., Tangermann M. (2011). Simulating the feel of brain-computer interfaces for design, development and social interaction, CHI 2011 Session Health 1: Technology Challenges, Vancouver, Canada.

Randolph A.B., Moore Jackson M.M. (2010). Assessing Fit of Non traditional Assistive Technologies, ACM Transactions on Accessible Computing, vol. 2, $\mathrm{n}^{\circ} 4$.

Schreuder M., Riccio A., Risetti M., Dähne S., Ramsay A., Williamson J., Mattia D., Tangermann M. (2013). User-centered design in brain-computer interfaces- A case study. Artificial Intelligence in Medecine, vol. 59, p. 71-80.

Sellers E.W., Vaughan T.M., Wolpaw J.R. (2010). A brain-computer interface for longterm independent home use. Amyotrophic Lateral Sclerosis, vol. 11, n5, p. 449-455.

Sepulveda F., Dyson M., Gan J.Q., Tsui C.S.L. (2007). A comparison of mental task combinations for asynchronous EEG-based BCIs, Proceedings of the 29th Annual International Conference of the IEEE EMBS 2007, Lyon, France, p. 23-26.

Silvoni S., Volpato C., Cavinato M., Marchetti M., Priftis K., Merico A., Tonin P., Koutsikos K., Beverina F., Piccione F. (2009). P300-based brain-computer interface communication evaluation and follow-up in amyotrophic lateral sclerosis, Frontier in Neurosciences, vol. $3, \mathrm{n}^{\circ} 60$.

Stone N.J., English A.J. (1998). Task type, posters, and workspace color on mood, satisfaction, and performance, Journal of Environmental Psychology, vol. 18, n², p. 175185

Tamburrini G. (2009). Brain to Computer Communication: Ethical Perspectives on Interaction Models, Neuroethics, vol. 2, p. 137-149.

Tan D.S., Nijholt A. (2010). Brain-Computer Interfaces and Human Computer Interaction, Applying our Minds to Human-Computer Interaction, D.S. Tan and A. Nijholt eds, Human-Computer Interaction Series, Springer, p. 3-19.

Trejo L.J., Rosipal R., Matthews B. (2006). Brain-Computer Interfaces for 1-D and 2-D Cursor Control: Designs Using Volitional Control of the EEG Spectrum or Steady-State Visual Evoked Potentials, IEEE transactions on Neural Systems and Rehabilitation Engineering, vol. 14, n 2, p. 125-129.

Van Hoof J., Mazej M., Hensen J.L.M. (2010). Thermal comfort: research and practice, Frontiers in Bioscience, vol. 15, n 2, p. 765-788. 
Van Langhenhove A., Bekaert M.-H., Nguyen J.-P. (2008). Using a brain-computer interface for rehabilitation: a case study on a patient with implanted electrodes, $4^{\text {th }}$ International BCI Workshop, 2008, Graz (Austria).

Vaughan T.M., McFarland D.J., Schalk G., Sarnacki W.A., Krusienski D.J., Sellers E.W., Wolpaw J.R. (2006). The Wadsworth BCI Research and Development Program: at home with BCI, IEEE Transactions on Neural Systems and Rehabilitation Engineering, vol. 14, n'2, p. 229-33.

Veitch, J.A., Newsham G.R., Boyce P.R., Jones C.C. (2008). Lighting appraisal, wellbeing and performance in open-plan offices: A linked mechanisms approach, Lighting Research \& Technology, vol. 4,0 n 2, p. 133-151.

Velliste M., Perel S., Spalding M.C., Whitford A.S., Schwartz A.B. (2008). Cortical control of a prosthetic arm for self-feeding, Nature, vol. 453, p. 1098-1101.

Wolpaw J., Birbaumer N., McFarland D., Pfurtscheller G., Vaughan T. (2002). Braincomputer interfaces for communication and control, Clinical Neurophysiology, vol.113, p. 767-791. 